Original Article

\title{
Effects of mental practice combined with electromyogram-triggered electrical stimulation for upper extremity function in stroke patients
}

\author{
Ji-Su Park ${ }^{1)}$, Jong-Bae Chol ${ }^{2)}$, Duk-Hyun An ${ }^{3)}$, Moon-Young Chang, OT, PhD ${ }^{4}{ }^{*}$ \\ 1) Department of Rehabilitation Science, Graduate School of Inje University, Republic of Korea \\ 2) Department of Occupational Therapy, Kyunghee Medical Center, Republic of Korea \\ 3) Department of Physical Therapy, Inje University, Republic of Korea \\ 4) Department of Occupational Therapy, Inje University: 197 Inje-ro, Gimhae, Gyeongsangnam-do \\ 621-749, Republic of Korea
}

\begin{abstract}
Purpose] To investigate the effect of mental practice combined with electromyogram-triggered electrical stimulation (MP-EMG ES) on the upper extremity of stroke patients. [Subjects and Methods] Participants were randomly assigned to experimental group or control group. The experimental group received MP-EMG ES plus conventional rehabilitation therapy for 5 days per week for 4 weeks. The control group received only conventional rehabilitation therapy. Outcome measure included the Fugl-Meyer Assessment (FMA) and Motor Activity Log (MAL). [Results] Experimental group showed more improved in the FMA, MAL-AOU, MAL-QOM compared with the control group. [Conclusion] These results suggest that MP-EMG ES improves the upper extremity of subacute stroke patients better than conventional rehabilitation therapy alone.

Key words: Electromyogram-triggered electrical stimulation, Mental practice, Stroke
\end{abstract}

(This article was submitted Jun. 9, 2017, and was accepted Jul. 14, 2017)

\section{INTRODUCTION}

Recently, several studies reported an intervention combining mental practice with electromyography-triggered electrical stimulation (MP-EMG ES) ${ }^{1,2}$. In MP-EMG ES, minute electrical signals are produced in the paretic upper extremity only through motor imagery without any actual movement of the upper extremity, and the EMG built into the instrument detects this and then provides electrical stimulation to the paretic upper extremity ${ }^{1}$. In other words, this treatment presents a new method combining mental practice and electrical stimulation, which have already been proven to be effective when applied separately ${ }^{3,4)}$. However, in the previous study, evidence of the combined treatment's effect on upper extremity function in patients following stroke remained unclear. Therefore, in the present study aimed to investigate the effects of the MP-EMG ES treatment on upper extremity function in patients with hemiparesis following a stroke.

\section{SUBJECTS AND METHODS}

In total, 40 patients were allocated into either the experimental $(n=16)$ or the control group $(n=16)$. Inclusion criteria were (1) had a first stroke with right or left hemisphere lesion, (2) Mini-Mental Status Examination score $>24$, (3) ability to imagination (an average score $<3$ on the vividness of Movement Imagery Questionnaire), and (4) 2 grades $<$ Modified Ashworth Scale. Exclusion criteria were the following: (1) implanted electronic devices including cardiac pacemakers or defibrillators, (2) skin lesion of affected side, (3) had history of seizure or epilepsy, and (4) unstable medical conditions. Inje

*Corresponding author. Moon-Young Chang (E-mail: myot@inje.ac.kr)

(C2017 The Society of Physical Therapy Science. Published by IPEC Inc.

(c) (1) $\odot$ This is an open-access article distributed under the terms of the Creative Commons Attribution Non-Commercial No Deriva-

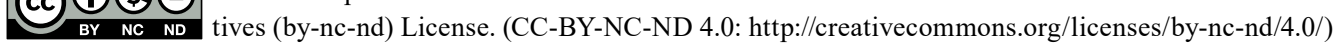


university ethics committee approved the study, and all participants provided informed, written consent prior to involvement in the study (2-1041024-AB-N-01-20150609-HR-251).

The experimental group received MP EMG-ES using mentamove (Mentamove Deutschland, Munich, Germany) and conventional rehabilitation therapy (CRT) whereas the control group received only CRT. CRT consisted of occupational and physical therapy, which included strength, ADL, balance, gait, coordination training. To apply experimental group on surface electrodes were attached on the wrist extensor muscle and reference electrode was attached at lateral side of forearm ${ }^{1)}$. The mentamove was in a three stages: (1) Motor imagery stage (approximately $12 \mathrm{sec}$ ), (2) electrical stimulation (approximately 6 $\mathrm{sec}$ ), and (3) relaxation (approximately $12 \mathrm{sec}$ ). Mentamove activates the muscle with its own biphasic waveform with pulse width ranging between 100 and $400 \mu \mathrm{S}$. Experimental group received the MP EMG-ES for 30 minutes/day, 5 days/week, for 4 weeks. The control group received only CRT, which was comparable to total training time received by experimental group. Upper extremity was measured by the Fugl-Meyer Assessment (FMA) and Motor Activity Log (MAL). The upper extremity subtest of the FMA was used as the changes of motor function ${ }^{5,6)}$. All statistical analyses were performed with SPSS 15.0 software (SPSS Inc., Chicago, USA). To evaluate the intervention effects, the paired t-test was used to compare measures pre and post the intervention in each group. The independent t-test was used to compare the changes in outcome measures between the 2 groups. Significance level was set at $p<0.05$.

\section{RESULTS}

The experimental group showed significant improvements from $21.69 \pm 5.80$ to $34.19 \pm 7.82$ for the FMA, and from $0.95 \pm 0.33$ to $2.43 \pm 0.51,0.99 \pm 0.38$ to $2.67 \pm 0.46$ for the MAL (AOU and QOM). The control group showed significant improvements from $23.31 \pm 5.65$ to $28.00 \pm 7.72$ for the FMA, and from $1.07 \pm 0.37$ to $1.79 \pm 0.62,1.07 \pm 0.35$ to $1.74 \pm 0.75$ for the MAL (AOU and QOM). After intervention, statistical analysis showed significantly different in FMA, MAL-AOU, and MAL-QOM (all, $\mathrm{p}<0.05)$ between groups.

\section{DISCUSSION}

Findings of the present study demonstrate that MP-EMG ES was more effective than CRT alone in improvement the upper extremity function of stroke patients. MP-EMG ES is to form sensorimotor circuits for movement through a repeated cycle of brain activation-signal transmission-muscle stimulation-movement and to use this for producing functional changes in the central nervous system and the body ${ }^{7}$. Hong et al. ${ }^{1}$ applied MP-EMG ES to patients in the stroke of chronic phase for 4 weeks. They found an increase in glucose metabolism in the supplementary motor, precentral, and postcentral gyri of the contralesional hemisphere, confirming the effectiveness of this treatment on brain activation. Moreover, Page et al. ${ }^{2)}$ applied MP-EMG ES to 5 patients with moderate upper extremity paralysis in the chronic phase of stroke, and found improved dexterity in the upper extremity and increased participation in physical activity. This is consistent with the results of our study, which provides evidence of the therapeutic effects of MP-EMG ES. We demonstrated the positive effects of MP-EMG ES on subacute stroke with hemiplegia better than CRT only. This study provides evidence to support the effects of MP-EMG ES in stroke patients compared with the control group.

\section{ACKNOWLEDGEMENT}

This work was supported by the 2016 Inje University research grant.

\section{REFERENCES}

1) Hong IK, Choi JB, Lee JH: Cortical changes after mental imagery training combined with electromyography-triggered electrical stimulation in patients with chronic stroke. Stroke, 2012, 43: 2506-2509. [Medline] [CrossRef]

2) Page SJ, Levine P, Hill V: Mental practice--triggered electrical stimulation in chronic, moderate, upper-extremity hemiparesis after stroke. Am J Occup Ther, 2015, 69: 1-8. [Medline]

3) Bustamante C, Brevis F, Canales S, et al.: Effect of functional electrical stimulation on the proprioception, motor function of the paretic upper limb, and patient quality of life: A case report. J Hand Ther, 2016, 29: 507-514. [Medline] [CrossRef]

4) Grabherr L, Jola C, Berra G, et al.: Motor imagery training improves precision of an upper limb movement in patients with hemiparesis. NeuroRehabilitation, 2015, 36: 157-166. [Medline]

5) Lee YY, Hsieh YW, Wu CY, et al.: Proximal fugl-meyer assessment scores predict clinically important upper limb improvement after 3 stroke rehabilitative interventions. Arch Phys Med Rehabil, 2015, 96: 2137-2144. [Medline] [CrossRef]

6) Hammer AM, Lindmark B: Responsiveness and validity of the Motor Activity Log in patients during the subacute phase after stroke. Disabil Rehabil, 2010, 32: 1184-1193. [Medline] [CrossRef]

7) Park JS, Choi JB, Kim WJ, et al.: Effects of combining mental practice with electromyogram-triggered electrical stimulation for stroke patients with unilateral neglect. J Phys Ther Sci, 2015, 27: 3499-3501. [Medline] [CrossRef] 